Commun. Korean Math. Soc. 26 (2011), No. 4, pp. 603-610

http://dx.doi.org/10.4134/CKMS.2011.26.4.603

\title{
AN IDENTIFICATION OF THE FREQUENCIES AND AMPLITUDES OF THE TRIGONOMETRIC SERIES
}

\author{
Ji Chan Chung, Min Soo Kang, Soo Han Kim, and Il Seog Ko
}

ABSTRACT. In this paper, we propose an algorithm for identifying $\omega_{j} \in$ $(0, \infty), a_{j}, b_{j} \in \mathbb{C}$ and $N$ of the following trigonometric series

$$
f(t)=a_{0}+\sum_{j=1}^{N}\left[a_{j} \cos \omega_{j} t+b_{j} \sin \omega_{j} t\right]
$$

by means of the finite number of sample values. We prove that the frequency components are shown to be the solutions of some characteristic equation related to the inverse of a Hankel matrix derived from the sample values.

\section{Introduction}

In this paper we consider the problem of identifying $\omega_{j} \in(0, \infty), a_{j}, b_{j} \in \mathbb{C}$ and $N$ of the following trigonometric series

$$
f(t)=a_{0}+\sum_{j=1}^{N}\left[a_{j} \cos \omega_{j} t+b_{j} \sin \omega_{j} t\right]
$$

by means of the finite number of values $f\left(t_{1}\right), \ldots, f\left(t_{L}\right)$ where the number $L$ of the values depends on $N$.

In engineering, it is well known that a (sound) signal can be represented as a trigonometric series as in (1). The algorithm developed in this paper thus can be applied to analyze the signals from the engineering point of view.

The main idea of this paper is originated from the paper [1] and [2] by El Badia and Ha-Duong. In those papers, the authors established an algebraic algorithm to solve inverse source problems for elliptic equations in $2 \mathrm{D}$ and $3 \mathrm{D}$ whose source terms are assumed to be the combination of either monopoles or dipoles. Applying the concept of the reciprocity gap functional (see [1] and references therein), they reduce the inverse problem to a problem of solving the

Received July 6, 2010.

2010 Mathematics Subject Classification. Primary 42A15; Secondary 15B05.

Key words and phrases. trigonometric series, Hankel determinant, signal processing.

(C)2011 The Korean Mathematical Society 
following algebraic equation

$$
c_{n}=\sum_{j=1}^{M} \lambda_{j} \alpha_{j}^{n}, \quad n=0,1,2, \ldots,
$$

where $c_{n}$ are given and $\alpha_{j}$ and $\lambda_{j}$ are unknowns and provide an algorithm to solve the equation (2) using the method of Hankel matrix decomposition. Since the main problem of this paper also can be reduced to the problem of solving the equation (2) (see the next section), we applied a similar, but more systematic, method proposed in $[1,2]$, to provide a detection algorithm. Precisely, it is shown that the frequency components $\omega_{j}$ are the solutions of some characteristic equation related to the inverse of a Hankel matrix derived from the sample values $f\left(t_{1}\right), f\left(t_{2}\right), \ldots, f\left(t_{L}\right)$.

One of the point of this paper is to develop an algorithm without depending on any advanced knowledge of mathematics but on the mathematics of elementary level.

\section{Identification of frequencies and amplitudes of the signal}

Throughout this paper, we assume an a priori information: we know an upper bound $N_{0}$ of $N$ and $M_{0}$ of $\omega_{j}$ for each $j=1,2, \ldots, N$.

Let $f$ be a trigonometric series of the form given in (1) whose values $f(t)$ on the set

$$
A=\left\{t_{n}=\frac{n \pi}{M_{0}} \mid n=0, \pm 1, \pm 2, \ldots, \pm\left(2 N_{0}+1\right)\right\}
$$

where $M_{0}$ is an upper bound of the set $\left\{\omega_{1}, \ldots, \omega_{N}\right\}$ are assumed to be, $a$ priori known. We will propose an algorithm to identify $a_{j}, b_{j} \in \mathbb{C}, \omega_{j} \in \mathbb{R}$ and $N$ from the sample values $f\left(t_{n}\right), t_{n} \in A$.

It is easy to see that

$$
\begin{aligned}
f\left(t_{n}\right) & =a_{0}+\sum_{j=1}^{N}\left[a_{j} \cos \omega_{j} \frac{n \pi}{M_{0}}+b_{j} \sin \omega_{j} \frac{n \pi}{M_{0}}\right] \\
& =\sum_{j=0}^{N}\left[\lambda_{j} \alpha_{j}^{n}+\overline{\lambda_{j} \alpha_{j}^{n}}\right]
\end{aligned}
$$

for $n=0, \pm 1, \ldots, \pm\left(2 N_{0}+1\right)$, where

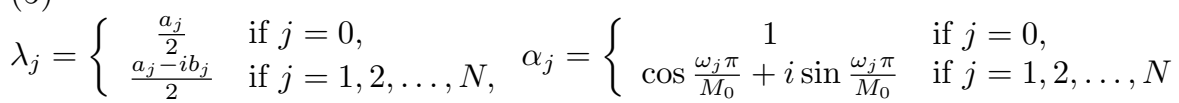

and $\bar{z}$ denotes the conjugate of $z \in \mathbb{C}$.

Define $c_{n}:=f\left(t_{-n}\right)+f\left(t_{n}\right), n=0,1, \ldots, 2 N_{0}+1$. Since $\overline{\alpha_{j}}=\alpha_{j}^{-1}$, we have

$$
c_{n}=\sum_{j=0}^{N}\left(\lambda_{j}+\overline{\lambda_{j}}\right)\left(\alpha_{j}^{n}+\overline{\alpha_{j}^{n}}\right), \quad n=0,1, \ldots, 2 N_{0}+1 .
$$


Lemma 2.1. For $\alpha \in \mathbb{C}$ with $|\alpha|=1,(\alpha+\bar{\alpha})^{n}=\sum_{k=0}^{\left[\frac{n}{2}\right]}\left(\begin{array}{c}n \\ k\end{array}\right)\left(\alpha^{n-2 k}+\right.$ $\left.\overline{\alpha^{n-2 k}}\right)$.

Proof.

$$
\begin{aligned}
& (\alpha+\bar{\alpha})^{n} \\
= & \sum_{k=0}^{n}\left(\begin{array}{l}
n \\
k
\end{array}\right) \alpha^{n-k} \overline{\alpha^{k}} \\
= & \frac{1}{2}\left[\sum_{k=0}^{n}\left(\begin{array}{l}
n \\
k
\end{array}\right) \alpha^{n-k} \overline{\alpha^{k}}+\sum_{k=0}^{n}\left(\begin{array}{l}
n \\
k
\end{array}\right) \overline{\alpha^{n-k}} \alpha^{k}\right] \\
= & \frac{1}{2}\left[\sum_{k=0}^{n}\left(\begin{array}{l}
n \\
k
\end{array}\right)\left(\alpha^{n-k} \overline{\alpha^{k}}+\overline{\alpha^{n-k}} \alpha^{k}\right)\right] \quad \\
= & \frac{1}{2}\left[\sum_{k=0}^{\left[\frac{n}{2}\right]}\left(\begin{array}{l}
n \\
k
\end{array}\right) \alpha^{k} \overline{\alpha^{k}}\left(\alpha^{n-2 k}+\overline{\alpha^{n-2 k}}\right)+\sum_{k=\left[\frac{n}{2}\right]+1}^{n}\left(\begin{array}{l}
n \\
k
\end{array}\right) \alpha^{n-k} \overline{\alpha^{n-k}}\left(\overline{\alpha^{2 k-n}}+\alpha^{2 k-n}\right)\right] \\
= & \sum_{k=0}^{\left[\frac{n}{2}\right]} C(n, k)\left(\alpha^{n-2 k}+\overline{\alpha^{n-2 k}}\right),
\end{aligned}
$$

where

$$
C(n, k)=\left\{\begin{array}{cc}
2\left(\begin{array}{l}
n \\
k
\end{array}\right) & \text { if } 2 k=n \\
\left(\begin{array}{l}
n \\
k
\end{array}\right) & \text { otherwise }
\end{array}\right.
$$

Remark 2.2. Following the above calculation, one can also prove that

$$
(\alpha-\bar{\alpha})^{n}=\sum_{k=0}^{\left[\frac{n}{2}\right]} C(n, k)(-1)^{k}\left(\alpha^{n-2 k}+(-1)^{n} \overline{\alpha^{n-2 k}}\right) .
$$

Define

$$
d_{n}:=\sum_{j=0}^{N} \mu_{j} \beta_{j}^{n}, \quad n=0,1, \ldots, 2 N_{0}+1,
$$

where $\mu_{j}=\lambda_{j}+\overline{\lambda_{j}}$ and $\beta_{j}=\alpha_{j}+\overline{\alpha_{j}}$. Then it follows from the above lemma that $d_{n}=\sum_{k=0}^{\left[\frac{n}{2}\right]} C(n, k) \cdot c_{n-2 k}$. Due to the above observation, we are led to the problems of

- detecting $N$ and

- solving the following system of equation

$$
d_{n}=\sum_{j=0}^{N} \mu_{j} \beta_{j}^{n}, \quad n=0,1, \ldots, 2 N_{0}
$$


for given $d_{0}, d_{1}, \ldots, d_{2 N_{0}+1}$, where $\mu_{j}$ and $\beta_{j}, j=0,1, \ldots, N$ are unknowns.

The above two problems can be solved based on the following simple observation.

Theorem 2.3. Let $d_{n}:=\sum_{j=0}^{N} \mu_{j} \beta_{j}^{n}, n=0,1, \ldots, 2 N_{0}$. For the following Hankel matrix of the size $n \times n$

$$
H_{n}:=\left(\begin{array}{cccc}
d_{0} & d_{1} & \cdots & d_{n-1} \\
d_{1} & d_{2} & \cdots & d_{n} \\
\vdots & \vdots & \ddots & \vdots \\
d_{n-1} & d_{n} & \cdots & d_{2 n-2}
\end{array}\right), \quad n=2,3, \ldots
$$

we have the following.

(i) $\operatorname{det} H_{N+1}=\mu_{0} \mu_{1} \cdots \mu_{N} \prod_{\substack{i, j=0 \\ i<j}}^{N}\left(\beta_{i}-\beta_{j}\right)^{2}$.

(ii) $\operatorname{det} H_{N+k}=0, \quad k=2,3, \ldots$

(iii) $l_{1}, l_{2}, \ldots, l_{N+1}$ satisfy

$$
x^{N+1}+l_{1} x^{N}+\cdots+l_{N} x+l_{N+1}=\left(x-\beta_{0}\right)\left(x-\beta_{1}\right) \cdots\left(x-\beta_{N}\right)
$$

if and only if $l_{1}, l_{2}, \ldots, l_{N+1}$ satisfy

$$
\left(\begin{array}{cccc}
d_{0} & d_{1} & \cdots & d_{N} \\
d_{1} & d_{2} & \cdots & d_{N+1} \\
\vdots & \vdots & \ddots & \vdots \\
d_{N} & d_{N+1} & \cdots & d_{2 N}
\end{array}\right)\left(\begin{array}{c}
l_{N+1} \\
l_{N} \\
\vdots \\
l_{1}
\end{array}\right)=\left(\begin{array}{c}
-d_{N+1} \\
-d_{N+2} \\
\vdots \\
-d_{2 N+1}
\end{array}\right)
$$

Proof. (i) can be easily proved from the following decomposition:

$$
H_{N+1}=\left(\begin{array}{cccc}
1 & 1 & \cdots & 1 \\
\beta_{0} & \beta_{1} & \cdots & \beta_{N} \\
\vdots & \vdots & \ddots & \vdots \\
\beta_{0}^{N} & \beta_{1}^{N} & \cdots & \beta_{N}^{N}
\end{array}\right)\left(\begin{array}{cccc}
\mu_{0} & 0 & \cdots & 0 \\
0 & \mu_{1} & \cdots & 0 \\
\vdots & \vdots & \ddots & \vdots \\
0 & 0 & \cdots & \mu_{N}
\end{array}\right)\left(\begin{array}{cccc}
1 & 1 & \cdots & 1 \\
\beta_{0} & \beta_{1} & \cdots & \beta_{N} \\
\vdots & \vdots & \ddots & \vdots \\
\beta_{0}^{N} & \beta_{1}^{N} & \cdots & \beta_{N}^{N}
\end{array}\right)^{T}
$$

Computing [the $(N+k)$ th column] $-l_{1} \times[$ the $(N+k-1)$ th column- $\cdots-$ $l_{N+1} \times[$ the $(k-1)$ th column], we have

$$
\operatorname{det}\left(\begin{array}{cccc}
d_{0} & d_{1} & \cdots & d_{N+k-1} \\
d_{1} & d_{2} & \cdots & d_{N+k} \\
\vdots & \vdots & \ddots & \vdots \\
d_{N+k-1} & d_{N+k} & \cdots & d_{2 N+2 k-2}
\end{array}\right)=\operatorname{det}\left(\begin{array}{ccccc}
d_{0} & d_{1} & \cdots & d_{N+k-2} & 0 \\
d_{1} & d_{2} & \cdots & d_{N+k-1} & 0 \\
\vdots & \vdots & \ddots & \vdots & \vdots \\
d_{N+k-1} & d_{N+k} & \cdots & d_{2 N+2 k-1} & 0
\end{array}\right)
$$

which proves (ii). Now let us prove the necessity of (iii). Suppose $l_{1}, \ldots, l_{N+1}$ be constants satisfying (8). Then it follows from the following decomposition 


$$
\begin{aligned}
& \left(\begin{array}{cccc}
d_{0} & d_{1} & \cdots & d_{N+1} \\
d_{1} & d_{2} & \cdots & d_{N+2} \\
\vdots & \vdots & \ddots & \vdots \\
d_{N} & d_{N+1} & \cdots & d_{2 N+1}
\end{array}\right) \\
= & \left(\begin{array}{cccc}
1 & 1 & \cdots & 1 \\
\beta_{0} & \beta_{1} & \cdots & \beta_{N} \\
\vdots & \vdots & \ddots & \vdots \\
\beta_{0}^{N} & \beta_{1}^{N} & \cdots & \beta_{N}^{N}
\end{array}\right)\left(\begin{array}{cccc}
\mu_{0} & 0 & \cdots & 0 \\
0 & \mu_{1} & \cdots & 0 \\
\vdots & \vdots & \ddots & \vdots \\
0 & 0 & \cdots & \mu_{N}
\end{array}\right)\left(\begin{array}{cccc}
1 & \beta_{0} & \cdots & \beta_{0}^{N+1} \\
1 & \beta_{1} & \cdots & \beta_{1}^{N+1} \\
\vdots & \vdots & \ddots & \vdots \\
1 & \beta_{N} & \cdots & \beta_{N}^{N+1}
\end{array}\right)
\end{aligned}
$$

that

$$
\left(\begin{array}{cccc}
d_{0} & d_{1} & \cdots & d_{N+1} \\
d_{1} & d_{2} & \cdots & d_{N+2} \\
\vdots & \vdots & \ddots & \vdots \\
d_{N} & d_{N+1} & \cdots & d_{2 N+1}
\end{array}\right)\left(\begin{array}{c}
l_{N+1} \\
l_{N} \\
\vdots \\
l_{1} \\
1
\end{array}\right)=\left(\begin{array}{c}
0 \\
0 \\
\vdots \\
0
\end{array}\right)
$$

which is equivalent to the equation (9). The sufficiecy of (iii) follows easily from the fact that $H_{N+1}$ is invertible.

The above theorem suggests that if we know the number $N$, then we first solve the equation (9) to find $l_{1}, l_{2}, \ldots, l_{N+1}$. We then solve the equation

$$
x^{N+1}+l_{1} x^{N}+\cdots+l_{N} x+l_{N+1}=0
$$

whose solutions are $\beta_{0}, \beta_{1}, \ldots, \beta_{N} \cdot \mu_{0}, \mu_{1}, \ldots, \mu_{N}$ can be calculated by using the decomposition (10). In order to determine $N$, we use the formula (i) and (ii) in Theorem 2.3. One starts with the upper bound $N_{0}$ of $N$ and compute the determinant of $H_{N_{0}+1}, H_{N_{0}}, H_{N_{0}-1}, \ldots$ until one first meet $H_{k+1}$ whose determinant is not zero. Then $k$ is the number $N$.

\section{Algorithm}

Based on the properties we have discussed in the previous section, we provide an algorithm.

\section{Algorithm.}

Given $f(t)=a_{0}+\sum_{j=1}^{N}\left[a_{j} \cos \omega_{j} t+b_{j} \sin \omega_{j} t\right]$, suppose the values $f(t)$ on the set

$$
A=\left\{t_{n}=\frac{n \pi}{M_{0}} \mid n=0, \pm 1, \ldots, \pm\left(2 N_{0}+1\right)\right\}
$$

are known. Let $c_{n}=f\left(t_{-n}\right)+f\left(t_{n}\right), n=0,1, \ldots, 2 N_{0}+1$.

[STEP I]

$$
\text { Compute } d_{n}=\sum_{k=0}^{\left[\frac{n}{2}\right]}\left(\begin{array}{c}
n \\
k
\end{array}\right) c_{n-2 k}, n=0,1, \ldots, 2 N_{0}+1 .
$$

[STEP II] 
Starting with $n=N_{0}$, compute the determinant $H_{n+1}$ for $n=N_{0}, N_{0}-1, \ldots$ until one first finds an integer $K$ satisfying $\operatorname{det} H_{K+1} \neq 0$. Let $N=K$.

[STEP III]

Solve the equation

$$
\left(\begin{array}{cccc}
d_{0} & d_{1} & \cdots & d_{N} \\
d_{1} & d_{2} & \cdots & d_{N+1} \\
\vdots & \vdots & \ddots & \vdots \\
d_{N} & d_{N+1} & \cdots & d_{2 N}
\end{array}\right)\left(\begin{array}{c}
l_{N+1} \\
l_{N} \\
\vdots \\
l_{1}
\end{array}\right)=\left(\begin{array}{c}
-d_{N+1} \\
-d_{N+2} \\
\vdots \\
-d_{2 N+1}
\end{array}\right)
$$

to calculate $l_{1}, l_{2}, \cdots, l_{N+1}$.

[STEP IV]

Solve the polynomial equation

$$
x^{N+1}+l_{1} x^{N}+\cdots+l_{N} x+l_{N+1}=0
$$

to obtain $N+1$ simple roots $\beta_{0}, \beta_{1}, \ldots, \beta_{N}$. From (5) and (7), we have $\beta_{j}=$ $2 \cos \frac{\omega_{j} \pi}{M_{0}}, j=1,2, \ldots, N$. Since $0 \leq \frac{\omega_{j} \pi}{M_{0}} \leq \pi, j=1,2, \ldots, N, \omega_{1}, \omega_{2}, \ldots, \omega_{N}$ are determined uniquely.

[STEP V]

Using the decomposition (10), calculate $\mu_{0}, \mu_{1}, \ldots, \mu_{N}$. Then it follows from (5) and (7) that $\mu_{j}=a_{j}, j=0,1, \ldots, N$.

[STEP VI]

To determine $b_{j}$, use the fact that

$$
f\left(t_{-n}\right)-f\left(t_{n}\right)=\sum_{j=1}^{N}\left(\lambda_{j}-\overline{\lambda_{j}}\right)\left(\alpha_{j}^{n}+\overline{\alpha_{j}^{n}}\right),
$$

where $\lambda_{j}=\frac{a_{j}-i b_{j}}{2}$.

Remark 3.1. Since (4) can be rewritten as

$$
f\left(t_{n}\right)=\sum_{j=0}^{4 N+1} \lambda_{j} \alpha_{j}^{n}
$$

with $\lambda_{N+j}:=\overline{\lambda_{j-1}}$ and $\alpha_{N+j}:=\overline{\alpha_{j-1}}$, it is also possible to apply the above algorithm directly from (12) (without using Lemma 2.1) to determine $\alpha_{j}$ and $\lambda_{j}$. But in this case, one have to calculate determinants and to solve homogeneous system of equations with respect to the Hankel matrices whose sizes are bigger that $(4 N+1) \times(4 N+1)$, which needs a great deal of amount of calculations comparing with the algorithm proposed in this paper.

\section{An application to the more general case}

In this section, we show that the method of this paper can be applied to identify the series whose form is more general than that of (1). 
Consider a series of the form

$$
f(t)=a_{0}+\sum_{j=1}^{N}\left[a_{j} \cos \tau_{j} t+b_{j} \sin \omega_{j} t\right],
$$

where the values $f\left(t_{n}\right), t_{n} \in A$ are a priori given. Here $A$ is the set defined the same as (3), where $M_{0}$ is an upper bound of the set $\left\{\tau_{j}, \omega_{j} \mid 1 \leq j \leq N\right\}$. Since

$$
\begin{aligned}
f\left(t_{n}\right) & =a_{0}+\sum_{j=1}^{N}\left[a_{j} \cos \tau_{j} \frac{n \pi}{M_{0}}+b_{j} \sin \omega_{j} \frac{n \pi}{M_{0}}\right] \\
& =\sum_{j=0}^{N}\left[\frac{a_{j}}{2}\left(\alpha_{j}^{n}+\overline{\alpha_{j}^{n}}\right)+\frac{b_{j}}{2 i}\left(\beta_{j}^{n}-\overline{\beta_{j}^{n}}\right)\right]
\end{aligned}
$$

for $n=0, \pm 1, \ldots, \pm\left(2 N_{0}+1\right)$, where

$$
\begin{aligned}
& \alpha_{j}=\left\{\begin{array}{cl}
1 & \text { if } j=0, \\
\cos \frac{\tau_{j} \pi}{M_{0}}+i \sin \frac{\tau_{j} \pi}{M_{0}} & \text { if } j=1,2, \ldots, N,
\end{array}\right. \\
& \beta_{j}=\left\{\begin{array}{cl}
1 & \text { if } j=0, \\
\cos \frac{\omega_{j} \pi}{M_{0}}+i \sin \frac{\omega_{j} \pi}{M_{0}} & \text { if } j=1,2, \ldots, N
\end{array}\right.
\end{aligned}
$$

and $b_{0}=0$. Define $c_{n}:=f\left(t_{-n}\right)+f\left(t_{n}\right)$ and $d_{n}:=f\left(t_{n}\right)-f\left(t_{-n}\right), n=$ $0,1, \ldots, 2 N_{0}+1$. We have

$$
c_{n}=\sum_{j=0}^{N} a_{j}\left(\alpha_{j}^{n}+{\overline{\alpha_{j}}}^{n}\right)
$$

and

$$
d_{n}=\sum_{j=0}^{N}-i b_{j}\left(\beta_{j}^{n}-{\overline{\beta_{j}}}^{n}\right) .
$$

Since $c_{n}$ is the same form as $(6), N, a_{j}$ and $\tau_{j}$ can be detected by following the same algorithm in the previous section. Now it remains to identify $b_{j}$ and $\omega_{j}$. Using the formula in Remark 2.2 instead of that in Lemma 2.1, one can easily modify the algorithm in the previous section to detect $b_{j}$ and $\omega_{j}$.

\section{References}

[1] A. El Badia and T. Ha-Duong, An inverse source problem in potential analysis, Inverse Problems 16 (2000), no. 3, 651-663.

[2] A. El Badia, Inverse source problem in an anisotropic medium by boundary measurements, Inverse Problems 21 (2005), no. 5, 1487-1506. 
Ji Chan Chung

Gyeonggi Science High School

Suwon 440-800, KoreA

E-mail address: jcchung93@hanmail.net

Min Soo Kang

HongChun High SchOOL

GyEONGGI-DO 449-150, KorEA

E-mail address: minsoo0707@naver.com

SOO HAN KIM

YUSHIN High SCHOOL

SuwON 442-190, KoreA

E-mail address: luckchris@naver.com

Il Seog Ko

Gyeonggi Science High School

Suwon 440-800, Korea

E-mail address: iskois@naver.com 